\title{
Representação Aberta e Semântica de Anotações Crowdsourcing em Mapas Web
}

\author{
André L. Gonzalez ${ }^{1}$, Diego Izidoro ${ }^{1}$, Roberto Willrich ${ }^{1}$, Celso A. S. Santos ${ }^{2}$ \\ ${ }^{1}$ Depto. de Informática e Estatística - Universidade Federal de Santa Catarina (UFSC) \\ Caixa Postal 476, 88040-900 - Florianópolis - SC - Brazil \\ ${ }^{2}$ Depto. de Informática - Universidade Federal do Espírito Santo (UFES) \\ Centro Tecnológico, CT VII, 29060-970 - Vitória - ES - Brazil \\ \{andre.lgonz, diego.izidoro, willrich\}@inf.ufsc.br, saibel@inf.ufes.br
}

\begin{abstract}
Nowadays, there is an increasing number of Web initiatives that rely on crowdsourcing via collaborative map annotations as problem-solving and data production models. Several of them allow users to annotate incidents of various kinds on a map, like crimes, crisis and health incidents. Ideally, these initiatives should produce open representation to enable people to share structured data and knowledge, and consequently improving mapping and problem-solving. This paper presents an approach for the production of open and semantic annotations of incidents on maps, allowing interoperability of both annotations and generated knowledge from these annotations. The proposed approach is performed and tested in our OurMap crowdsourcing system.
\end{abstract}

Resumo. Atualmente, é crescente o número de ferramentas Web que adotam crowdsourcing, via anotações colaborativas em mapas, como modelo de resolução de problemas e produção de dados. Várias delas permitem aos usuários anotar incidentes de diferentes tipos no mapa, como incidentes relacionados à segurança, saúde e transporte. Idealmente, estas iniciativas deveriam oferecer dados abertos, possibilitando o compartilhamento das anotações e do conhecimento gerado, com consequente melhoria no mapeamento e na resolução de incidentes. Este artigo propõe uma abordagem de produção de anotações abertas e semânticas de incidentes em Mapas, permitindo a interoperabilidade das anotações $e$ da semântica do conhecimento gerado a partir delas. A abordagem proposta é adotada e testada no nosso sistema crowdsourcing OurMap.

\section{Introdução}

Crowdsourcing é um modelo de produção para resolver problemas com base na inteligência e conhecimento coletivos [Brabham 2008]. Amazon Mechanical Turk, Waze, Foursquare e Wikipédia são exemplos de serviços ofertados na Web recentemente que se apoiam neste modelo. Algumas das principais vantagens do crowdsourcing são o custo relativamente baixo para a geração de soluções e a facilidade de resolução de problemas que demandam elevado grau de cognição, uma vez que se apoiam no trabalho humano [Pedersen 2013]. O modelo crowdsourcing também tem sido aplicado na construção colaborativa de mapas Web a partir da identificação de elementos geolocalizados nestas estruturas [Goodchild 2007]. O OpenStreetMap (OSM) [Haklay 2008] e o wikimapia.org são exemplos de sistemas de mapas que se apoiam no 
mapeamento voluntário de informações geográficas relacionadas a lugares imóveis e com um ciclo de vida longo, tais como ruas, cidades e construções, num processo denominado de Volunteered Geographic Information (VGI).

O foco deste artigo é a anotação colaborativa e voluntária de elementos em mapas Web para relato de incidentes. Diferentemente dos sistemas de VGI, o foco aqui é a anotação de elementos geolocalizados com um ciclo de vida mais curto. Este tipo de serviço já é atualmente oferecido por sistemas Web especializados para alguns domínios, tais como crimes [Shah 2011, Furtado 2010], crises [Ushahidi 2013] e saúde [Qureshi 2011]. Esses sistemas oferecem recursos para qualquer usuário anotar um mapa para informar a ocorrência de um incidente observado/conhecido por ele em uma região específica. Assim, esses sistemas permitem capturar o conhecimento coletivo dos seus usuários sobre determinado domínio, com informações de localização, categorização e descrição dos incidentes. Tal conhecimento pode ser utilizado como forma de apoio a tomada de decisão pelos gestores públicos ou outras organizações. Por exemplo, em situações de crises (inundações, terremotos, incêndios, etc.), o sistema pode apoiar o mapeamento das regiões em crise e a geração de respostas a estas situações críticas. Os sistemas atuais de anotação de incidentes em mapas tem algumas limitações que são tratadas neste trabalho, como a falta de representação formalizada do conhecimento coletivo, a especialização a apenas um certo domínio (p.e., crise, saúde, crimes), e muitos geram anotações que não são interoperáveis com outros sistemas.

A maioria dos sistemas de anotações de incidentes em mapas produz anotações fechadas sobre seus mapas, que não podem ser reutilizadas fora deles, em outros sistemas de mapas. Um esforço para permitir a produção de anotações interoperáveis está sendo proposto pelo grupo W3C Open Annotation, por meio da padronização do modelo Anotações Abertas (OA - Open Annotation) [W3Ca 2013]. Este modelo utiliza RDF e segue os princípios de Dados Interligados (LD - Linked Data) [Berners-Lee 2006]. As anotações, representadas sob a forma de dados abertos, tornam possível o reuso destas informações por outras iniciativas, aumentando a capacidade de mapear e de resolver incidentes a partir de conteúdo adicionado aos mapas.

Ontologias são especificações formais (passíveis de interpretação por computadores) de conceitos, utilizadas para descrever um domínio de interesse [Fonseca 2012]. Conceitos, ou classes, são representações da informação modelada, e possuem propriedades e relações com outros conceitos. A adoção de abordagens semânticas oferece diversas vantagens aos sistemas de anotação em mapas. Uma primeira vantagem é o fato de que os sistemas poderiam ser mais flexíveis, em termos de domínio de aplicação. Através da importação de ontologias de domínio, um sistema poderia facilmente se adaptar a um determinado domínio. Outra vantagem refere-se à possibilidade dos usuários do sistema categorizar o tipo de incidente, identificar a localização e descrever o incidente (quando ocorreu e uma descrição textual). Com ontologias, é possível não apenas oferecer meios de categorizar os incidentes, mas permitir a associação de propriedades aos incidentes (p.e., relações com estimativa de valores em assaltos, número de pessoas envolvidas, temperatura, dimensões físicas, etc.) e relações com outros conceitos (proximidade com locais, relações com outros incidentes, etc.). Todas estas propriedades e relações poderiam ser "aprendidas" pelo sistema graças à importação da ontologia.

GONZALEZ, A. L.; IZIDORO, D.; WILLRICH, R.; SANTOS, C. A. S.

Representação Aberta e Semântica de Anotações Crowdsourcing em Mapas Web

iSys - Revista Brasileira de Sistemas de Informação, Rio de Janeiro, vol. 7, No. 2, p. 48-66, 2014. 
O uso de abordagens semânticas ainda possibilita uma melhoria na qualidade da informação gerada pelos usuários, um problema bem conhecido dos sistemas que se apoiam no crowdsourcing [Goodchild 2010]. Por exemplo, a ocorrência de um buraco na via pública no meio do mar ou uma infecção hospitalar em um ponto que não seja próximo a um hospital são exemplos de anotações inconsistentes com relação à localização de um incidente. Estas inconsistências podem ser evitadas com a formalização do conhecimento de domínio. No estudo de caso da Seção 5, a ontologia utilizada obriga que as anotações sobre incidentes em um linha de ônibus estejam sempre relacionadas, em termos de localização, a uma rua ou avenida pertencente ao percurso desse ônibus.

A representação formalizada do conhecimento coletivo com base em ontologias oferece outras vantagens, como a realização de buscas semânticas e o uso de inferências. No primeiro caso, os usuários podem expressar critérios de busca com base em conceitos e propriedades, especificadas nas ontologias, importantes na geração de relatórios consolidados que darão suporte à tomada de decisão. Um exemplo é a identificação do número de assaltos ocorridos em cada bairro de uma cidade. Neste caso, a inferência poderia ser usada para gerar novos conhecimentos a partir das anotações, como a categorização das localizações de incidentes de acordo com seus tipos e quantidades anotados. Cabe ressaltar que o suporte à buscas semânticas suportadas e à geração de conhecimento por inferência é independente do domínio ao qual o sistema de anotação em mapas está inserido.

Este artigo propõe uma abordagem de produção de anotações abertas e semânticas de incidentes em mapas Web. A representação de anotações proposta segue o modelo OA e permite que anotações tenham como alvo não apenas recursos na Web, mas também coordenadas geográficas, referenciadas segundo o esquema proposto por meio do URI geo [Mayrhofer 2010]. A representação aberta de anotações proposta permite criar dados ligados (LD - Linked Data) que poderiam ser reutilizados por qualquer aplicação, mesmo com uso de diferentes serviços de mapa Web, como o OSM e o Google Maps [Google Maps API 2014].

$\mathrm{Na}$ abordagem proposta, as anotações em mapas podem ser marcadas semanticamente pela associação da anotação a indivíduos mantidos em uma Base de Conhecimento (BC) Ontológica. A diferença entre ontologias e $\mathrm{BC}$ é a seguinte: ontologias definem um esquema (ou modelo de dados) que representa os conceitos dentro de um domínio e suas propriedades (atributos e relacionamentos). BC utiliza conceitos, definidos pelas ontologias, para descrever uma determinada realidade, através da inclusão de indivíduos. Na presente proposta, os próprios usuários podem colaborar na população da $\mathrm{BC}$ com indivíduos que descrevem semanticamente lugares e incidentes. A linguagem de representação do conhecimento adotada é a OWL [OWL 2004]. Dessa forma, tanto as anotações em mapas quanto as informações geradas seguem representações abertas. A BC pode ser usada para recuperação de informação e descoberta de conhecimento baseado em ontologias, de forma a facilitar a tomada de decisões.

Existem algumas ontologias para informações geográficas [Couclelis, 2010] e para eventos [Gangemi, 2002], [Raimond, 2007], [van Hage, 2011]. Após uma análise das ontologias existentes, não foi localizada uma ontologia genérica o suficiente que represente conceitos básicos relacionados às anotações de incidentes geolocalizados. 
Por isso, foi definida a ontologia de alto nível denominada OurMap para a marcação semântica dos incidentes anotados.

As principais contribuições da abordagem proposta neste artigo, em relação às existentes, são: (i) a utilização do esquema OA para as anotações, tornando-as independentes de sistemas de mapas Web; (ii) a possibilidade de anotar diretamente localizações geográficas, por meio da utilização do URI geo e (iii) a geração de uma base de conhecimento ontológica que formaliza a representação da informação gerada de forma colaborativa, verificando a consistência das relações espaciais de acordo com a classe de objeto anotado.

Após esta introdução, o texto está organizado em outras cinco seções. A Seção 2 apresenta uma contextualização por meio de conceitos sobre ontologias geolocalizadas e de incidentes e representação aberta. A Seção 3 descreve os requisitos de sistemas de anotação de incidentes em mapas e analisa os principais trabalhos relacionados. $\mathrm{Na}$ Seção 4 é apresentada a contribuição deste artigo, a abordagem de representação aberta de anotações semânticas de incidentes em mapas usando o modelo OA. A Seção 5 apresenta uma prova de conceito da proposta dentro do sistema OurMap, implementado pelos autores. Finalmente, as conclusões e trabalhos futuros são apresentados na Seção 6 , seguida pela lista de referências citadas no texto.

\section{Contextualização}

Esta seção introduz alguns temas relevantes ao entendimento da proposta, a saber, ontologias de informações geolocalizadas e de incidentes, bem como a representação de anotações abertas.

\subsection{Ontologias de Informações Geolocalizadas e de Eventos}

Existem diversos trabalhos apontando as vantagens do uso de abordagens baseadas em ontologias no domínio de Sistemas de Informações Geográficas (GIS - Geographical Information System), como [Couclelis 2010], [Fonseca 2002] e [Arpinar 2004]. Dentre estas vantagens, pode-se citar a possibilidade de integrar, compartilhar e analisar informações geoespaciais.

Diversas ontologias propostas para GIS têm por objetivo especificar conceitos geoespaciais [Zhao 2009, Sen 2012]. Estas ontologias especificam conceitos no domínio de GIS, possibilitando a aplicação destes em sistemas que se utilizam do conceito de crowdsourcing para a construção de mapas cartográficos (crowdsourced maps).

Em outros domínios que fazem uso de métodos de crowdsourcing para indicação de incidentes em mapas, por exemplo, nas áreas de desastres e de apoio à gestão pública, as ontologias GIS não são adequadas. Crowdsourcing baseado em interfaces de mapas Web exigem conceitos mais amplos associados à geopolítica (com conceitos de entidades político-administrativas, como países, cidades, bairros, estradas e vias). Algumas ontologias, tal como a ontologia geopolítica desenvolvida pela FAO (Food and Agriculture Organization of the United Nations) [FAO 2012], não cobrem todos os conceitos necessários para a produção crowdsourcing de informações sobre problemas urbanos diversos. Para tanto, é necessário abranger todos os conceitos relacionados ao local onde a população pode associar algum dado, na forma de uma anotação digital. 
Neste artigo foi adotada a definição de incidente e problema definida pela ITIL (Information Technology Infrastructure Library) [ITIL, 2014]: Incidente é um evento que não é parte da operação padrão de um serviço e que pode causar uma interrupção do serviço ou uma redução de sua qualidade; e Problema é uma condição com frequência identificada como resultado de múltiplos incidentes.

Um alto grau de interoperabilidade semântica em sistemas de anotações de incidentes em mapas requer a adoção de ontologias de alto nível, permitindo que o conhecimento possa ser compartilhado entre diferentes plataformas de anotações em mapas. Existem algumas iniciativas de estabelecimento de uma ontologia de alto nível que têm potencial para serem usadas no contexto de anotação de incidentes em mapas. As principais iniciativas propõem ontologias de alto nível para eventos (um termo mais geral que incidente) e são analisadas detalhadamente pelos trabalhos de [Shaw 2009] e [Van Hage 2011]. Estas ontologias diferem quanto à definição de características temporais do evento e de suas propriedades.

\subsection{Anotações Digitais Abertas}

Algumas tentativas para estabelecer a padronização de representação de anotações de recursos digitais foram propostas nos últimos anos, destacando-se aquelas realizadas pelos projetos Annotea [Kahan 2001], Annotation Ontology [Ciccarese 2011] e Open Annotation Collaboration (OAC) [Haslhofer 2011]. Atualmente, o grupo W3C Open Annotation Community [W3Ca 2013] está propondo a conciliação destas duas últimas propostas, através da especificação do modelo OA. O presente trabalho adotou o modelo OA para representação aberta das anotações geradas.

O modelo OA, baseado em RDF e seguindo os princípios de LDs, oferece um framework interoperável e extensível para expressar anotações em recursos digitais de modo que elas possam ser facilmente compartilhadas entre plataformas. Este modelo oferece uma riqueza de expressão suficiente para satisfazer requisitos complexos e, ao mesmo tempo, manter-se suficientemente simples, permitindo seu uso em casos menos complicados. Para isso, está sendo desenvolvido o Modelo de Dados de Núcleo (OA) [Sanderson 2013], um namespace que define um conjunto de classes de base do modelo e os aspectos diretamente relacionados com a arquitetura Web.

As classes definidas pelo Modelo de Dados de Núcleo são: oa:Annotation, uma especificação, na forma de grafo serializado, de uma relação entre dois ou mais recursos; oa:Target é o alvo da anotação, o que está sendo anotado; e oa:Body é o corpo de uma anotação, que comenta o recurso ou relaciona outro recurso ao alvo.

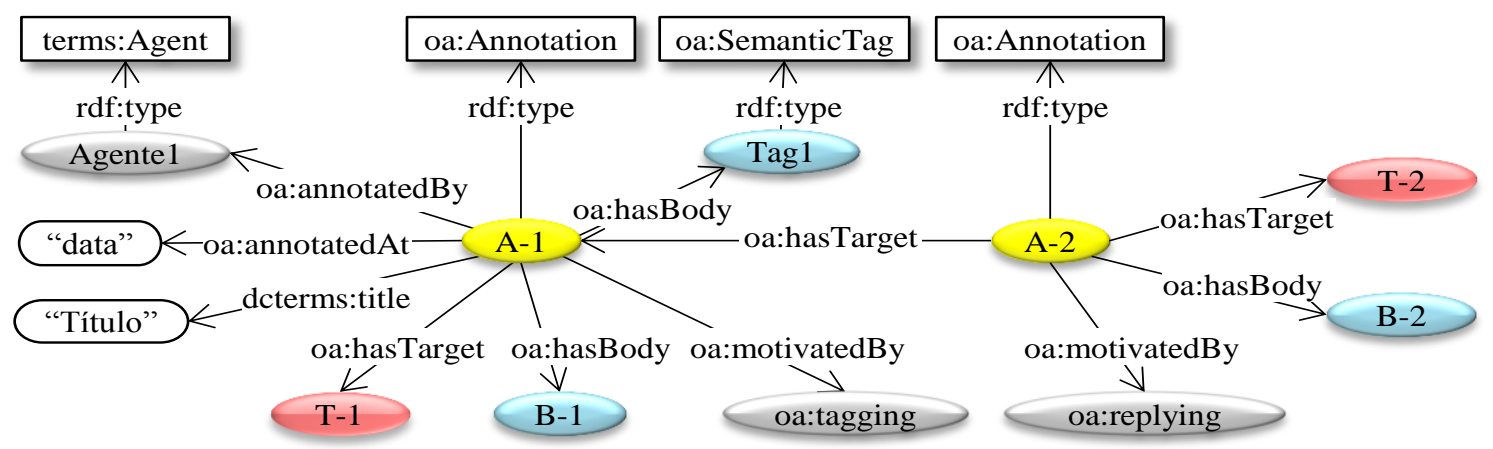

Figura 1. Anotações A-1 e A-2 e sua representação no modelo OA. 
A Figura 1 apresenta duas anotações digitais (A-1 e A-2) representadas no modelo OA. A anotação A-1 é representada por um oa:Annotation e tem como alvo T-1 (oa:Target), referenciado pela propriedade oa:hasTarget. O corpo da anotação A-1 é formado por dois indivíduos referenciados pela propriedade oa:hasBody, sendo B-1 (Body) um recurso qualquer sobre T-1, e Tag1, um indivíduo do tipo oa:SemanticTag, que adiciona um valor semântico à anotação. Além de corpo e alvo, a anotação tem várias propriedades, como autor (oa:annotator), título (dcterms:title) e data de criação (oa:annotatedAt). É recomendado que a anotação tenha a relação oa:motivatedBy com uma instância da classe oa:Motivation, possibilitando uma descrição mais completa e precisa sobre a razão da criação de cada anotação. A Anotação A-2 é um exemplo de anotação de resposta a A-1, ilustrando como o OA pode representar debates em fóruns de discussão.

\section{Anotações de Incidentes em Mapas}

Esta seção identifica alguns requisitos importantes para sistemas Web que oferecem recursos para anotações colaborativas de incidentes em mapas. Também são avaliados os principais trabalhos relacionados quanto ao atendimento destes requisitos.

O primeiro requisito é o oferecimento de dados abertos. Por exemplo, adotando o modelo OA, uma iniciativa de crowdsourcing pode gerar dados que podem ser reusados por outras iniciativas. Apesar de oferecerem dados abertos, as iniciativas OSM, wikimapia.org e GeoNames [GeoNames, 2014] não permitem a anotação em mapas de "coisas" que tenham uma vida relativamente curta e que tenham um posicionamento espacial pouco preciso, como é o caso de muitos incidentes. A grande maioria das iniciativas de anotação de incidentes em mapas não adota representação aberta. Uma exceção é PublicSafetyMap.org, que oferece os dados em RDF/XML. Além desta, na área de anotação de mapas históricos, MapHub [MapHub 2013] segue o modelo OA.

Sistemas de Mapas Web, como OSM e Google Maps, são destinados à identificação de lugares imóveis e com vida longa, com uma localização fixa e conhecida, como ruas ou construções civis. Contrariamente, incidentes e/ou problemas possuem características bem diferentes, de forma que as anotações associadas a ambos devem levar em conta:

- Características temporais: o anotador de um incidente pode saber o instante ou o intervalo de tempo no qual o incidente ocorreu, ou pode não saber quando o incidente teve início ou quando terminou. Ele simplesmente pode tê-lo observado em um dado momento. Portanto, na anotação de um incidente, o sistema deve prover flexibilidade em termos de posicionamento temporal do incidente.

- Características de localização: diferentemente de um local geográfico imóvel, muitos incidentes podem não ter uma localização muito bem definida ou conhecida. O sistema deve permitir a descrição da localização não apenas por coordenadas geográficas precisas, mas também por relações espaciais genéricas [Klien 2005], como à esquerda de, à direita de, atrás, em frente, próximo, dentro e fora. 
- Informações Temáticas: para ser mais preciso e útil, o relato de um incidente deve definir o tipo ou categoria de incidente. Para tal, devem ser definidos vocabulários de tipos de incidentes dependentes do domínio de aplicação.

Em termos de características espaciais, as ferramentas analisadas pelos autores permitem ao anotador expressar a localização do incidente de maneira precisa, na forma de coordenadas geográficas ou regiões bem definidas (como coordenadas geográficas, ou endereço contendo nome da rua, número, bairro). Esta forma de localização pode, muitas vezes, não representar o local preciso do incidente, podendo levar a conclusões erradas. Considerando agora as características temporais, alguns sistemas, como PortoAlegre.cc [PortoAlegre.cc 2013], não permitem expressar em que momento ocorreu a causa relatada. Por sua vez, as plataformas Ushahidi [Ushahidi 2013] e Wikicrimes [Furtado 2010] permitem especificar data e horário do incidente relatado, mas ambas utilizam o conceito de intervalo temporal.

Outro requisito importante é a garantia de interoperabilidade do conhecimento gerado a partir dos relatos dos usuários. Para isso, é importante a adoção de abordagens semânticas, que podem contribuir em três aspectos [Kim 2008]: representação robusta das entidades e seus relacionamentos; facilitação da troca de conhecimento; e ontologias que permitem representar a semântica dos dados de um modo que eles possam ser processáveis por máquina, possibilitando a análise de dados e reconhecimento de conceitos, para processos de inferência e busca semântica, permitindo a obtenção de resultados mais amplos e precisos [Lamas 2008].

Diferentemente de algumas iniciativas de produção de VGI, poucos sistemas de anotação de incidentes em mapas oferecem recursos de marcação semântica. Em geral, estas ferramentas adotam categorias de incidentes (informações temáticas) que não seguem a estruturação de uma ontologia para armazenar seus dados, tornando a interpretação e reutilização de seus conteúdos mais difíceis. Não há nem mesmo a definição de uma terminologia comum, podendo ser usados termos diversos, como relato, evento, causa ou anotação, para se referenciar ao que é anotado no mapa.

O trabalho de [Schulz 2012] é uma das poucas aplicações em que o vocabulário de tipos de incidente com descrição semântica utiliza RDFS (RDF Schema), no qual cada tipo é definido como uma classe. Além deste, a ferramenta MapHub, embora não se destine à anotação de incidentes, oferece recursos para marcação semântica das anotações, usando referências a conceitos definidos por sistemas como [DBpedia, 2014] e [GeoNames, 2014].

\section{Representação de Anotações Digitais Abertas e Semânticas em Mapas}

O modelo OA é um framework de representação aberta de anotação em recursos digitais. Para sua aplicação em anotações em mapas é necessário definir uma representação que permita a especificação de alvos de anotação, de forma a não apenas anotar lugares nomeados, mas também localizações geoespaciais. As vantagens de utilizar uma representação aberta são as mesmas de qualquer dado aberto. Elas são particularmente importantes para iniciativas que utilizam o modelo crowdsourcing para geração de dados e resolução de problemas. Como as anotações são abertas, aquelas geradas por uma iniciativa podem ser reusadas por outras, mesmo quando as iniciativas utilizam sistemas de mapas diferentes para suporte às anotações. 
No caso de anotações sobre lugares nomeados no modelo OA, normalmente os alvos das anotações são URIs, que especificam os lugares utilizando, por exemplo, serviços como GeoNames ou DBPedia. Em uma situação hipotética na qual um usuário desejasse fazer uma anotação digital sobre a cidade de Florianópolis-SC, ele poderia utilizar como alvo o URI http://sws.geonames.org/3463237/, que identifica, de forma única, esta cidade no GeoNames. Assim, a localização geográfica de uma entidade geoespacial é especificada como uma propriedade da mesma usando, por exemplo, o vocabulário WGS84 [WGS84 1984].

A subseção 4.1 a seguir detalha a abordagem proposta para representação aberta de anotações digitais estruturadas e semânticas em mapas sobre localizações geoespaciais e incidentes.

\subsection{Utilizando URIs geo em anotações abertas em mapas}

A especificação OA não explicita como representar anotações digitais sobre localizações geográficas de forma independente de sistemas Web. Isto é importante no caso de anotações em localizações não nomeadas. Em muitos casos de relatos de incidentes em sistemas de mapas Web, o alvo, da anotação, referenciado pelo anotador é a coordenada geoespacial onde ocorreu o incidente e não o posicionamento do incidente no sistema de mapas Web que está sendo usado por ele. Por exemplo, no caso de um relato de um buraco de rua próximo a uma coordenada específica, o sistema Web apresentando o mapa para o usuário deve ser considerado uma facilidade para $o$ anotador localizar a coordenada geográfica na qual o incidente ocorreu. Para ser uma anotação aberta e independente de sistemas de mapas Web, esta anotação deveria especificar um alvo (a coordenada geográfica) de maneira independente de qualquer fonte de dados Web (incluindo o próprio sistema de mapa Web utilizado pelo anotador).

A RFC 5870 [Mayrhofer 2010] padroniza um esquema de URI para localizações geográficas em duas ou três dimensões, o chamado URI geo. Este tipo de URI é compacto, simples, legível por humanos e independente de protocolos. É considerado um passo na direção de facilitar, suportar e padronizar o problema de identificação de localização em aplicações e serviços geoespaciais. Este esquema de URI dá suporte ao uso das coordenadas geográficas latitude, longitude e altitude, possibilitando que diferentes sistemas possam compartilhar suas informações e entender que, por exemplo, o URI geo: $-27.599217,-48.519018$ identifica uma localização física.

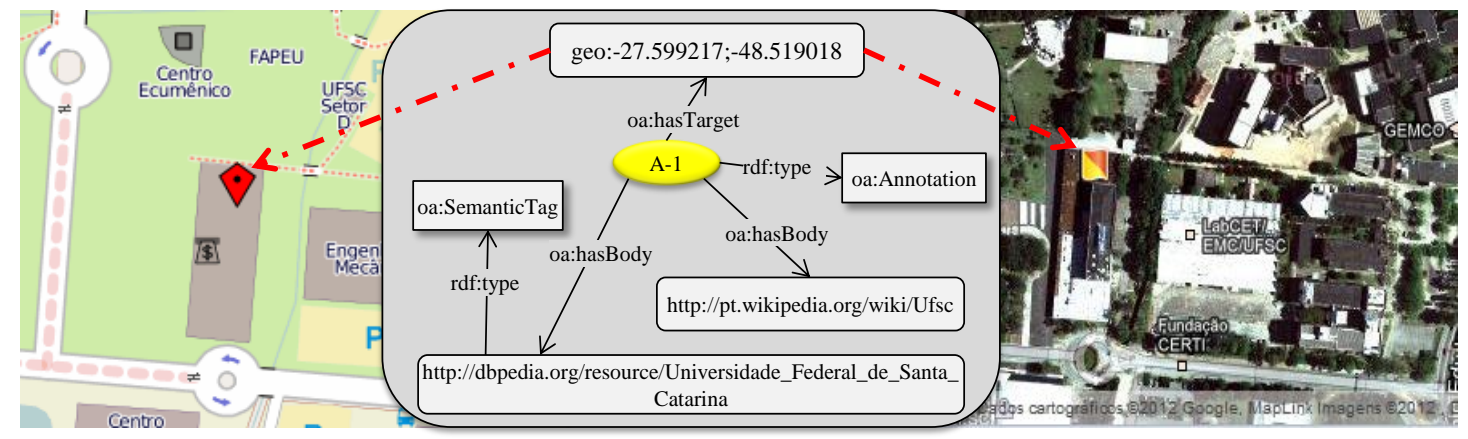

Figura 2. Anotações de coordenadas com o URI geo e o Modelo OA.

Como alvos de anotações no modelo OA são expressos por URI, o uso de URI geo permite produzir anotações digitais sobre localizações geográficas de maneira aberta e independente de sistemas de mapas Web. A Figura 2 ilustra o uso do modelo 
OA para representar uma anotação digital em localizações geográficas com o esquema de URI geo. Esta anotação tem como alvo (oa:hasTarget) o URI geo:-27.599217, 48.519018 (localização geográfica da reitoria da UFSC). O corpo da anotação (oa:hasBody) é a página desta universidade na Wikipédia. Esta anotação tem uma marcação semântica (oa:SematicTag) associando informação semântica à anotação, sendo utilizado o conjunto de dados da DBpedia.

As partes laterais da Figura 2 apresentam o mapeamento da posição geográfica marcada pela anotação nos sistemas de mapas Web OSM (esquerda) e Google Maps (direita). Para que esta abordagem seja possível, o sistema de anotação em mapas deve redirecionar a URI geo para o sistema de mapas Web adotado, de forma que anotações feitas em um sistema de mapas Web possam ser exibidas corretamente em qualquer outro sistema deste tipo.

\subsubsection{Ontologia de Incidentes proposta}

Uma representação de incidentes em mapas deveria considerar que as localizações espaciais e temporais de incidentes podem ser genéricas. Outro aspecto importante a ser considerado em sistemas de crowdsourcing é a dificuldade de garantir a qualidade da informação gerada voluntariamente pela comunidade [Goodchild 2010]. Uma abordagem semântica deveria garantir um mínimo de consistência semântica em termos de localização destas informações. Pela falta de uma ontologia genérica o suficiente para abranger os conceitos básicos relacionados às anotações de incidentes geolocalizados associados a elementos da vida em comunidade no contexto deste trabalho, foi definida a ontologia de alto nível OurMap.

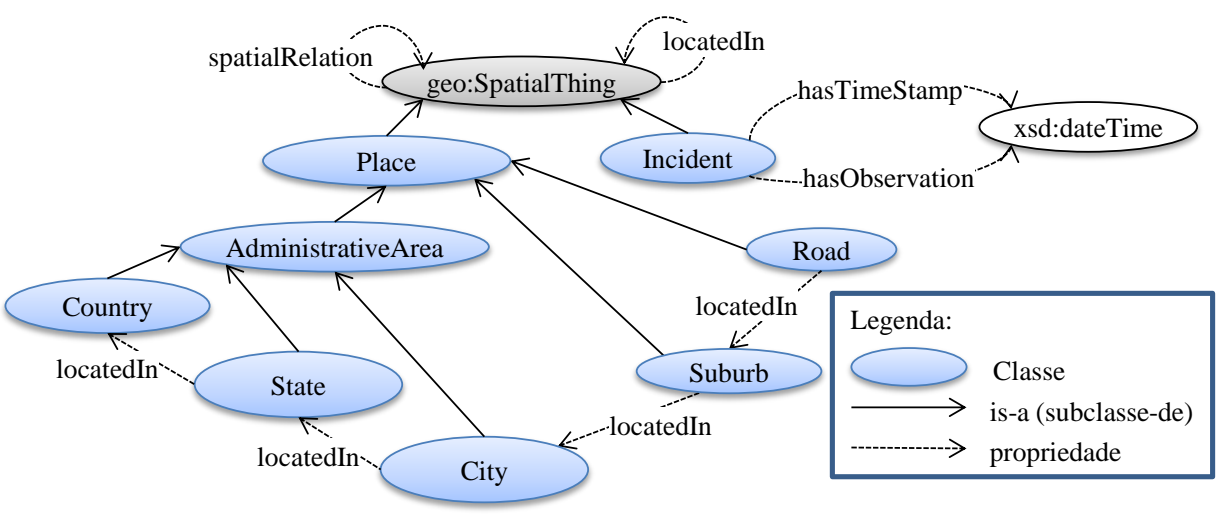

Figura 3. Visão simplificada da Ontologia OurMap.

Uma visão simplificada da ontologia OurMap, disponível em http://biblio.inf.ufsc.br/ spaces/ontology/ourmap.owl, é representada na Figura 3. As ontologias definem um conjunto de conceitos que descrevem um domínio. Os conceitos são descritos por meio de classes, onde uma classe é uma estrutura que abstrai um conjunto de indivíduos.

As duas principais classes da ontologia OurMap são Place e Incident, que são subclasses de geo:SpatialThing definida no vocabulário W3C Basic Geo [W3Ca 2003] de posicionamento geográfico. A classe http://schema.org/Place representa algo imóvel ou uma localização. A classe Incident representa um incidente, como definido pela ITIL (ver seção 3). A classe http://schema.org/AdministrativeArea é uma região geográfica sob jurisdição de um governo particular, possuindo como subclasses 
http://schema.org/Country, http://schema.org/State e http://schema.org/City. Outras duas subclasses (Suburb e Road) são definidas para a gestão de localizações relacionadas a cidades.

Propriedades são as relações a serem construídas entre as classes e seus indivíduos (relação binária) ou para o indivíduo (função, característica). A propriedade locatedIn da ontologia OurMap permite especificar relacionamentos de localização entre lugares. Note que as subclasses de Place podem ter subclasses de indivíduos que tenham relações locatedIn apenas com indivíduos SpatialThing particulares. Na ontologia OurMap define-se que indivíduos Road têm relação locatedIn com Suburb, este com City, e assim por diante. Isto garante um mínimo de consistência dos lugares administrativos, bairros e ruas.

Incident pode ter uma relação de localização (locatedIn) com qualquer outro SpatialThing. Além disso, um indivíduo da classe SpatialThing pode ter uma relação espacial genérica (spatialRelation) entre outros SpatialThing. Diversas sub-relações de spatialRelation foram definidas: under, isinside, encloses, near e over. Além dessas, mais seis sub-relações de near foram definidas: behind, beside, rightOf, leftOf, inFrontOf e adjacent. Por fim, foi definida uma sub-relação de adjacent chamada onTop. Nas suas especificações, foram definidas as características de simetria e transitividade destas propriedades, visando permitir a realização de inferências de proximidade entre anotações, como, por exemplo, afirmar que um Incident está relacionado, em termos de proximidade, a um Place.

As propriedades de dados hasTimeStamp e hasObservation da classe Incident definem, respectivamente, os instantes ou períodos de ocorrência e de observação de um incidente. Estas propriedades têm como tipo literal xsd:dateTime. hasTimeStamp é usada caso o incidente tenha seu instante ou intervalo de ocorrência conhecido. hasObservation é usada em casos em que os instantes inicial e/ou final do incidente sejam desconhecidos. hasTimeStamp define um instante e suas duas subpropriedades juntas (hasStart, hasFinish) definem um intervalo. Por sua vez, estas duas últimas possuem subpropriedades que permitem especificar intervalos de tempo imprecisos (hasStartAfter, hasStartBefore, hasFinishAfter, hasFinishBefore). Da mesma forma, hasObservation especifica o instante e suas duas subpropriedades (hasStartObservation e hasFinishObservation) permitem definir intervalos. Foram também definidas regras SWRL para atribuir valores às propriedades hasStartBefore e hasFinishAfter no caso de incidentes com instantes ou intervalos de tempo de observação. Estas regras expressam a consequência de que, caso o incidente seja observado num dado instante, o início do incidente é anterior a esse instante, e o fim é posterior.

\subsubsection{Definindo Categorias de Incidentes e Lugares}

Graças à possibilidade de reuso de ontologias existentes, pode-se estender a ontologia OurMap para adaptá-la a um domínio específico, definindo as categorias de incidentes ou lugares que se queira anotar. Por exemplo, se o OurMap for utilizado para a área de transporte público, pode-se importar uma ontologia de transporte público, como OTN [Lorenz 2005]. Se o foco for informação turística, pode-se importar uma ontologia de turismo, como e-tourism [Prantner, 2004] para a construção do sistema de anotação.

Também é possível definir restrições de localização das novas classes de lugares e incidentes, da mesma forma adotada para lugares administrativos. Por exemplo, caso 
seja importada a ontologia OTN, tem-se uma nova classe chamada Accident, que representa um acidente. Para adaptá-la à abordagem proposta, deve-se incluir a especificação de que Accident é uma subclasse de Incident, e que esta classe de indivíduo pode ter localização (locatedIn) Road. Novamente, esta operação permite controlar minimamente a semântica de localização dos acidentes (devem estar sempre em ruas ou avenidas).

Em termos de categorias de incidentes, a abordagem proposta permite, além da importação de categorias de incidentes preexistente, a especificação de uma hierarquia de subclasses Incident. Para cada subclasse, podem ser especificadas restrições quanto à localização de lugar. Por exemplo, uma subclasse de incidente Pothole (buraco na rua) poderia ser especificada de maneira que indivíduos desta classe devem ter uma relação de localização com uma rua.

\subsection{Populando Bases de Conhecimento a partir de Anotações Digitais}

As propostas de sistemas de anotações semânticas em mapas existentes suportam apenas a marcação semântica das anotações. No caso, um lugar ou um relato de incidente pode ser marcado semanticamente por um termo definido por uma entidade externa (como o DBpedia) e pelo próprio sistema Web, no caso do uso de RDF para categorização dos tipos de incidentes, como adotado em [Schulz 2012]. A presente proposta sugere o armazenamento do conhecimento gerado pelas anotações em uma base de conhecimento, na qual lugares e incidentes são representados como indivíduos. Os URIs destes indivíduos são usados como marcadores semânticos das anotações.

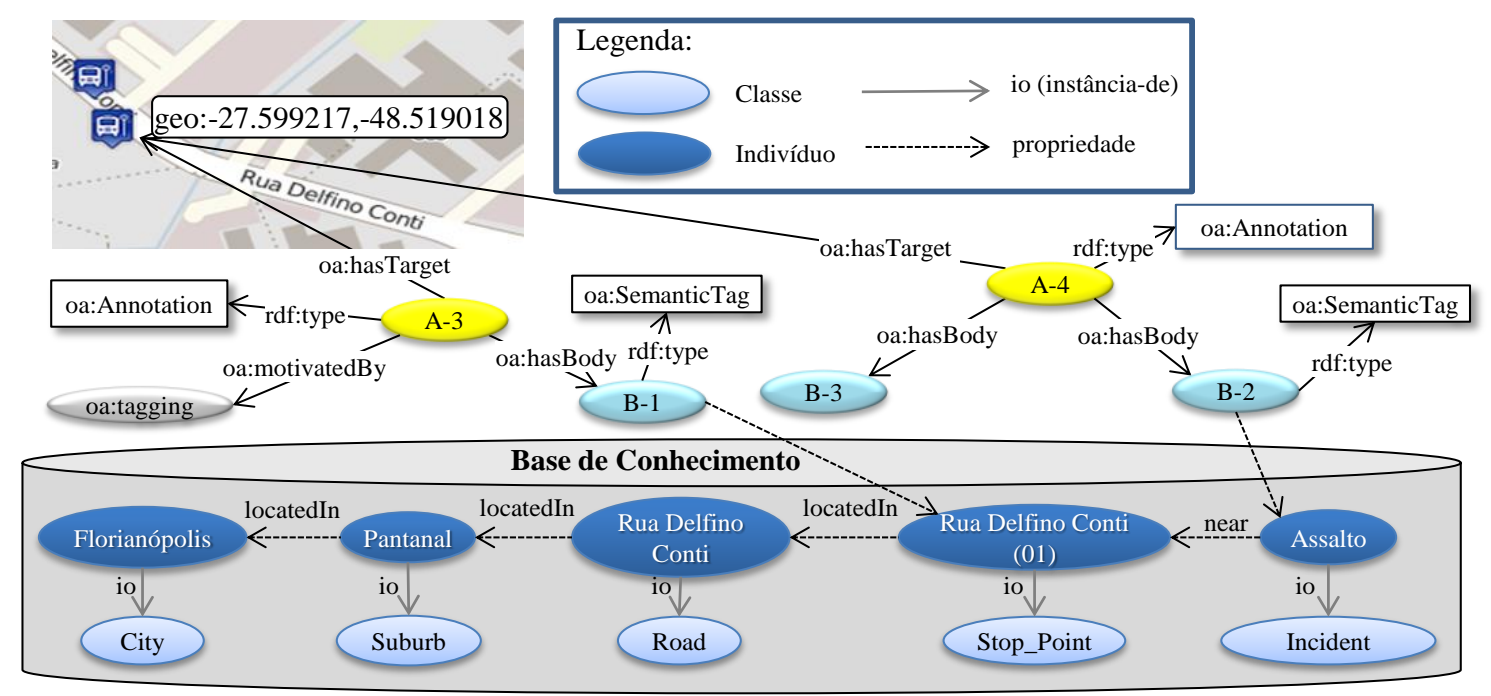

Figura 4. Anotações Semânticas e a Base de Conhecimento.

Para ilustrar esta população da base de conhecimento, considere as duas anotações apresentadas na Figura $4(\mathrm{~A}-3$ e $\mathrm{A}-4)$. Considere ainda que a base contenha todos os indivíduos que representam bairros e ruas da cidade de Florianópolis. A-3 é uma anotação gerada pelo usuário para anotar um ponto de ônibus em uma coordenada. Nesta operação ele também realiza a instanciação de um indivíduo Stop_Point na base de conhecimento. Note que esta anotação foi possível pois a localização geográfica geo:-27.599217,-48.519018 é próxima a uma rua (como especificado na ontologia), chamada Rua Delfino Conti, que está localizada no bairro Pantanal da 
cidade de Florianópolis. A-4 é uma anotação de um assalto próximo ao ponto de ônibus anotado por A-3. Essa relação de proximidade é definida por meio da relação near entre os indivíduos Assalto e Rua Delfino Conti (01).

Nas soluções de anotações em mapas existentes, seria associada uma tag semântica para indicar que A-3 é uma anotação sobre Stop_Point e A-4 é sobre assalto. A presente proposta marca as anotações com os indivíduos na base de conhecimento. Isso permite considerar os relacionamentos existentes entre os indivíduos (como definido na ontologia), e permite verificar restrições de localizações. Outras vantagens incluem a possibilidade de realização de buscas semânticas mais complexas e eficientes, além de permitir que inferências sejam feitas para gerar novo conhecimento.

\section{Visão Geral do Sistema OurMap}

Esta seção apresenta uma visão geral do sistema OurMap, o sistema de anotações em mapas que segue a abordagem de produção de anotações abertas e semânticas de incidentes em mapas proposta neste artigo.

A implementação do sistema OurMap foi feita em linguagem Java e a biblioteca OpenLayers [OpenLayers, 2014] e com o sistema de mapas OSM. Para o desenvolvimento dos aspectos semânticos, foi adotada a API Jena [Jena API 2013]. Um protótipo com funções mínimas foi implementado para permitir a realização de testes do tipo prova de conceito relacionados à geração e uso das anotações produzidas sobre um mapa.

\subsection{Personalizando o OurMap}

Por meio da interface de administração desenvolvida para o OurMap, o administrador pode personalizar o sistema para o(s) domínio(s) de incidentes desejado(s). Em um primeiro momento, é possível criar novas categorias de anotações de lugares e incidentes. Uma categoria de incidentes agrupa um conjunto de classes de Incident, de forma a facilitar a seleção do tipo de incidente ao qual se refere uma nova anotação. Essa seleção de categoria e classe de incidente é realizada pelo usuário anotador no momento da criação da anotação. Exemplos de categorias de incidentes incluem Segurança Pública e Transporte Público.

Após a criação das categorias, o administrador pode importar ontologias que descrevam lugares e incidentes de diferentes domínios. A importação de ontologias se dá por meio de funcionalidades providas pela API Jena, via a indicação do URL da ontologia. Por questões de desempenho, no momento da seleção das classes anotáveis, as informações sobre as classes anotáveis (nome e propriedades) são mantidas no banco de dados.

Em seguida, pode-se especificar as classes das ontologias importadas que serão passíveis de sofrer anotações pelos usuários (as classes às quais será permitida a instanciação de indivíduos na BC). A Figura 5 apresenta a interface de seleção de classes anotáveis de uma ontologia de domínio. As classes apresentadas nesta figura pertencem à ontologia OTN [Lorenz, 2005].

$\mathrm{Na}$ interface de seleção de classes anotáveis é possível especificar o ícone associado ao lugar ou incidente, os locais de ocorrência admissíveis para cada classe e à qual categoria pertence esta classe. Por exemplo, na Figura 5 é apresentada a definição de que a classe Stop_Point pertence a categoria de lugar Transporte Público, sendo que 
as áreas administrativas que admitem a localização de um Stop Point são Suburb e Road. É a partir desta interface, que o sistema define as categorias de incidente (classe Incident) e lugares (classes Places) como descrita na Seção 4.1.2.

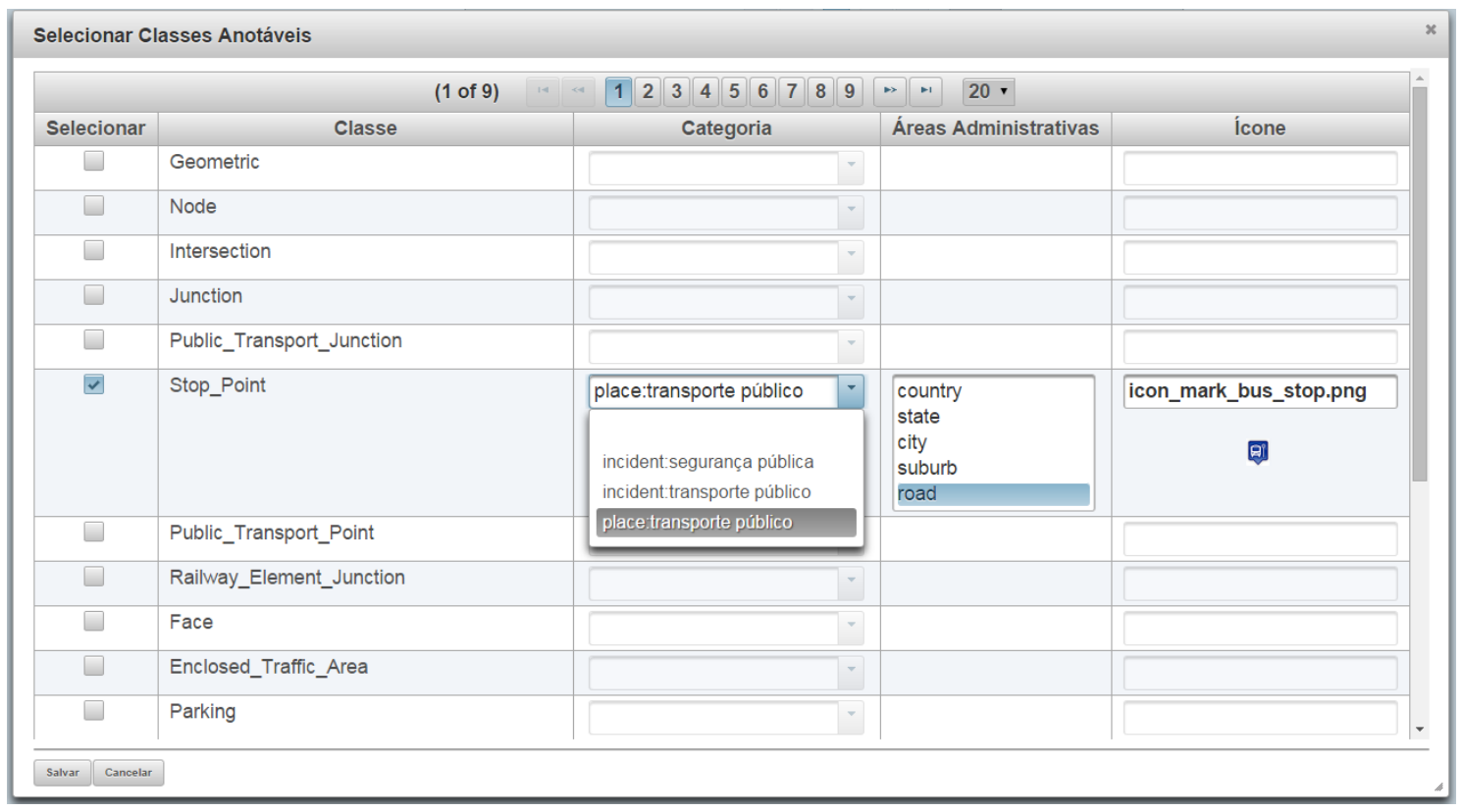

Figura 5. Especificando classes anotáveis.

\subsection{Inicializando a base de conhecimento}

Dependendo do cenário de utilização do OurMap, é necessário inicializar a Base de Conhecimento (BC) representando determinados indivíduos que podem sofrer anotações ou então indivíduos que tem relação com o indivíduo anotado.

Nesta implementação prova de conceito, o objetivo foi a anotação de incidentes na área urbana da cidade de Florianópolis-SC, em particular, anotações relacionadas ao transporte público. Para tal, é conveniente inicializar a $\mathrm{BC}$ com indivíduos representando a cidade de Florianópolis e todos os bairros, ruas e pontos de ônibus da cidade. A forma adotada para este procedimento foi o desenvolvimento de um script para a geração de indivíduos bairros, ruas e pontos de ônibus de Florianópolis na base de conhecimento a partir na base de dados do OSM.

Caso não houvesse a inicialização da $\mathrm{BC}$, os próprios usuários, por meio de anotações no mapa, seriam responsáveis por popular esta base. Por exemplo, no momento de anotar algum incidente em um ponto de ônibus, caso o ponto de ônibus não esteja ainda anotado no mapa, o anotador teria que criar a anotação no ponto, gerando o indivíduo ponto de ônibus na $\mathrm{BC}$, bem como indivíduos especificando a rua onde $\mathrm{o}$ ponto se localiza, e o bairro (caso estes ainda não estiverem representados na $\mathrm{BC}$ ). A inicialização da $\mathrm{BC}$ evita a geração destes indivíduos no momento da anotação do incidente.

\subsection{Gerando anotações na base de conhecimento}

As anotações digitais podem ser geradas de duas formas: (i) manual, pelos usuários do sistema OurMap, utilizando a Interface com o Usuário ou (ii) automática, pelo script de anotação, a partir dos dados abertos disponibilizados por outras iniciativas. 
Nesta implementação prova de conceito foram geradas automaticamente anotações sobre todos os pontos de ônibus da cidade de Florianópolis, a partir da base de dados do OSM. Para esta operação foi utilizado um script para a criação de anotações. As coordenadas e nomes dos pontos foram extraídos da base de dados do OSM.

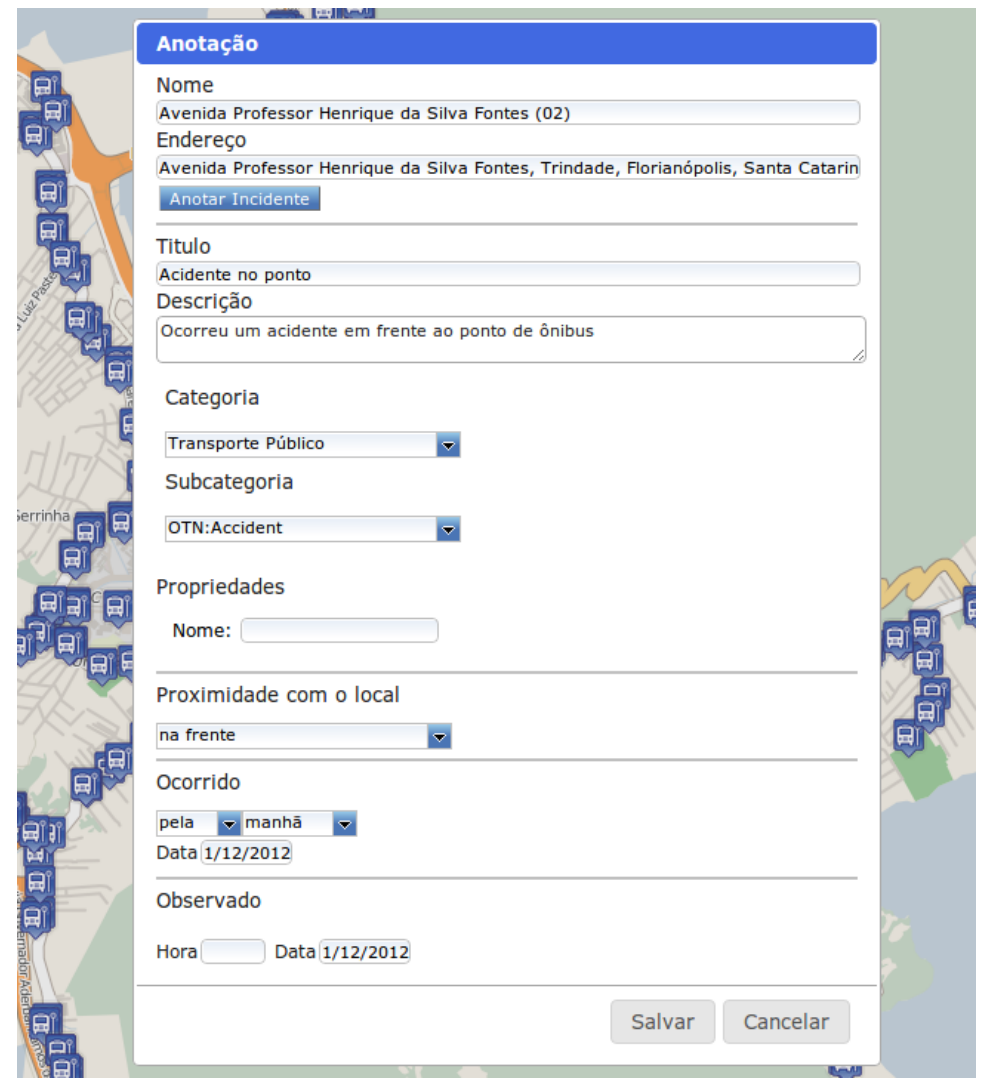

Figura 6. Interface de relato de incidente.

Para criar manualmente uma anotação de um lugar ou incidente, o usuário deve clicar sobre o mapa na localização onde ele deseja proceder a anotação, ou então sobre uma outra anotação (que representa um lugar ou incidente já anotado). A Figura 6 ilustra a criação de uma anotação de um incidente cujo referencial é um ponto de ônibus. Neste caso, para anotar o incidente, o usuário deve clicar sobre o ícone do ponto de ônibus no mapa, e em seguida anotar o incidente clicando no botão "Anotar Incidente" (visto na Figura 6).

A interface de criação de anotação, ilustrada pela Figura 6, apresenta os campos que o usuário poderá preencher para gerar a anotação. Inicialmente, ele deve selecionar a categoria de incidente e a sua subcategoria. A subcategoria se refere a classe de incidente da ontologia. Depois de selecionada a subcategoria, as propriedades (definidas pela ontologia) da classe são exibidas, e o usuário pode especificar seus valores. Note que nesta interface é possível determinar as características espaciais e temporais do incidente, por meio dos campos "Proximidade com o local" (espaço) e "Ocorrido" e "Observado" (tempo). 


\subsection{Tratamento de Problemas}

Não basta apenas que os usuários da Web relatem incidentes no mapa, é necessário que as entidades responsáveis deem uma resposta aos usuários que identificaram os incidentes. O termo tratamento de problema é utilizado aqui para referenciar todas as etapas que são necessárias para resolver, ou amenizar, o problema gerado pelo(s) incidente(s). Por exemplo, caso um usuário relate um "buraco em uma rua", é necessário que o poder público indique que tomou ciência do problema, que este será tratado e que o usuário que relatou o incidente seja comunicado sobre o status deste tratamento. O sistema OurMap está sendo desenvolvido também para atuar como um sistema de gerenciamento de incidentes anotados.

O administrador do sistema pode, em sua interface, determinar os papéis dos usuários cadastrados no OurMap. Dentre estes papéis está o de gerente. Um gerente é responsável por tratar certos tipos de incidentes relatados pelo sistema OurMap. O administrador pode definir área(s) geográfica(s) (país, estado, cidade, bairro ou rua) e/ou categoria(s) de incidentes pelas quais cada gerente será responsável.

No sistema OurMap, os incidentes possuem um estado de tratamento (novo, em tratamento ou resolvido). Sempre que o usuário relata um incidente, ele inicia com o estado novo. Em um pós-processamento da anotação, o sistema atribui o incidente relatado a um gerente, com base no local de ocorrência de incidente e na sua categoria. Quando o gerente acessa o sistema, ele tem à disposição uma interface para visualizar os incidentes que deve tratar, os incidentes em tratamento e os já resolvidos. O gerente pode utilizar a ferramenta de fórum de discussão existente em cada anotação para dar uma resposta a sociedade sobre o tratamento do incidente.

\subsection{Acessando os dados abertos}

Atualmente, o acesso aos dados é feito via uma API pública OurMap, oferecida por meio de um Serviço Web RESTful. Esta API permite consultas sobre as anotações existentes no Sistema OurMap. As informações apresentadas por estas consultas são armazenadas em um banco de dados MySQL.

A API pública OurMap suporta a especificação de parâmetros de consultas feitas por meio do URL da API. Os parâmetros são adicionados após o caractere “?”, e são inseridos sequencialmente, em qualquer ordem, separados por um caractere " $\&$ ", na forma "nome_do_parâmetro=valor". Os parâmetros disponíveis para especificar as consultas são:

- resp: formato de arquivo de resposta esperado (os formatos aceitos são RDF/XML, Turtle e Json);

- from: valor, identificador de anotação, inicial para um intervalo;

- to: valor, identificador de anotação, final para um intervalo;

- startTime: data, de criação de anotação, inicial para um intervalo;

- endTime: data, de criação de anotação, final para um intervalo;

- author: nome do usuário criador das anotações;

- annotableClass: nome da classe anotável que especifica as anotações.

Um exemplo de URL de consulta utilizando parâmetros é "http://biblio.inf.ufsc.br:8080/spaces/public/api/ws/annotation/oa?resp=xml\&start

Time=2013-06-03T00:00:00-03:00\&annotableClass=stop_point", o qual especifica o 
formato do arquivo de resposta como $\mathrm{RDF} / \mathrm{XML}$, e busca apenas anotações da classe stop_point criadas a partir de 03/06/2013.

Uma API de consulta por meio da linguagem de consultas RDF SPARQL (SPARQL Protocol and RDF Query Language) está em desenvolvimento. Esta linguagem permite recuperar e manipular informações armazenadas no formato RDF. Desta forma, o sistema OurMap permitirá que as informações referentes à anotações e à base de conhecimento sejam consultadas, assim sendo possível definir uma consulta SPARQL para que seu resultado seja o mais pertinente possível. Um exemplo seria uma consulta que recuperasse todas as ruas de uma determinada cidade.

A abordagem descrita permite a construção de buscas de mais alto nível, não sendo necessário que o usuário entre com as coordenadas da região da qual queira extrair informações. Estas buscas de alto nível podem ser feitas visando recuperar informações referentes a anotações ou dados da base de conhecimento. Para consultas referentes a anotações, pode-se utilizar uma busca SPARQL como:

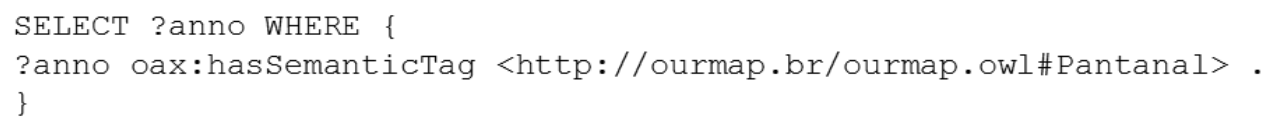

A consulta acima permite, por exemplo, recuperar as anotações que têm como alvo o bairro Pantanal. Seu resultado é uma lista de todas as anotações feitas sobre um local do mapa representado por coordenadas geográficas.

\section{Experimentação}

Conforme citado na seção 5, foi implementado um protótipo do sistema OurMap cujo objetivo é a realização de testes do tipo prova de conceito relacionados à geração e uso das anotações produzidas sobre um mapa. Nesta experimentação, o protótipo foi configurado para dar suporte a anotações de lugar e de incidente no domínio de transporte público. Para tal, foi importada a OTN [Lorenz 2005] que define conceitos relacionados a transporte público, tanto em termos de lugares (como Stop_Point, Parking, Airport) e incidentes (como Accident, Emergency). Seguindo o procedimento definido na Seção 5.1, esta ontologia foi importada para o OurMap. As classes desta ontologia passíveis de sofrerem anotações nos mapas foram definidas, sendo especificados os locais de admissíveis de suas ocorrências. Por exemplo, a classe Stop_point teve sua localização restrita a locais próximos a instâncias de Road.

Para testar a ontologia de incidentes OurMap, foram incialmente realizadas a população da BC com todos os bairros (instâncias de Suburb) e ruas (instancias de Road) da cidade de Florianópolis (instância de City). Estas informações foram obtidas da base de dados do OSM. Também foram obtidas desta base de dados todos os pontos de ônibus, que foram representados como instâncias de Stop_point na BC com relações de localizações a indivíduos de Road. Além de popular a BC, no caso de pontos de ônibus, foram geradas anotações de lugares Stop_Point sobre o mapa.

$\mathrm{Na}$ sequência, foram simuladas anotações de incidentes de transporte público, nas diversas classes anotáveis, sendo que as anotações permitiram associar valores às propriedades definidas nas classes, comprovando-se assim a capacidade de adaptação de OurMap ao domínio da ontologia importada. 
Consultas às anotações abertas OA foram realizadas através da API Pública do OurMap, de forma que as codificações RDF geradas foram validadas por meio do serviço de validação de RDF da W3C [W3Cb 2013].

\section{Conclusões}

O modelo de produção de dados geoespaciais em crowdsourcing já vem sendo utilizado por diversas comunidades, como forma de permitir a produção voluntária de informações por usuários finais sobre serviços a eles oferecidos. Este artigo propõe uma representação aberta de anotações digitais em mapas de incidentes seguindo os princípios de dados ligados. Esta representação é resultado da adoção do esquema OA proposto pelo W3C e uso do URI geo, para referenciar coordenadas geográficas independentemente de sistemas de mapas Web. Além disso, o conhecimento gerado pelas anotações digitais é mantido em bases de conhecimento ontológicas. Com a representação em OWL, podem-se realizar busca de informações e a descoberta de conhecimento baseada em ontologias, possibilitando uma melhor tomada de decisões.

O artigo também apresenta uma implementação prova de conceito do sistema OurMap que utiliza a representação aberta de anotações digitais proposta. Além disso, também adota a linguagem OWL para representação da base de conhecimento ontológica. Graças a isso, OurMap pode ter seu domínio de aplicação personalizado pela importação de ontologias de domínio. A base de conhecimento ontológica representa o conhecimento gerado de maneira voluntária pelos usuários do sistema. Com a representação em OWL, podem-se realizar buscas de informação e descoberta de conhecimento baseada em ontologias, melhorando assim a tomada de decisões.

Como trabalhos futuros, deve ser disponibilizada a consulta, através da API pública, por anotações filtradas pelas categorias. Além disso, deve ser finalizada e testada a API de consulta por meio de SPARQL, para então ser disponibilizada. Devem ser, também, feitos testes reais, com a utilização do sistema OurMap por vários usuários fazendo relatos de novos incidentes e consultando relatos existentes, além da gestão dos relatos por usuários gerentes. A importação das anotações por outros sistemas, visando verificar a flexibilidade da proposta, também é um trabalho futuro.

\section{Agradecimentos}

Este trabalho realizado no contexto do Projeto SPACES-4D: Sistema PArticipativo de Gestão e Monitoramento de Cidades e Serviços Públicos usando rastreamento com câmeras 4D, financiado pelo CTIC/RNP.

\section{Referências}

Arpinar, I. B., Sheth, A., Ramakrishnan, C. (2004) “Geospatial Ontology Development and Semantic Analytics". In Handbook of Geographic Information Science, Wilson, J.P., Fotheringham, A.S., Editors. 2004, Blackwell Publishing.

Berners-Lee, T. (2006) "Linked Data - Design Issues". http://www.w3.org/DesignIssues/LinkedData.html.

Brabham, D. C. (2008) "Crowdsourcing as a Model for Problem Solving: An Introduction and Cases". In Convergence: The Int. Journal of Research into New Media Technologies 14(1):75-90. 
Ciccarese, P. et al. (2011) "An Open Annotation Ontology for Science on Web 3.0". In Journal of Biomedical Semantics, 2(Suppl 2): S4.

Couclelis, H. (2010) "Ontologies of geographic information". In Intl. Journal of GIS, 24(12), 2010. DOI=10.1080/13658816.2010.484392.

DBPedia (2014) http://dbpedia.org.

DC-Terms (2012). http://purl.org/dc/terms/.

Dublin Core (2012). http://purl.org/dc/elements/1.1/.

FAO (2012) Food and Agriculture Organization of the United Nations http://www.fao.org/

Fonseca, F. T. (2002) "Using Ontologies for Integrated Geographic Information Systems". In Transactions in GIS, 6, 2002.

Furtado, V. et al. (2010) "Collective intelligence in law enforcement - The WikiCrimes system”. In Information Sciences, 180(1): 4-17.

Gangemi, N. et al. (2002) "Sweetening Ontologies with DOLCE". In Knowledge engineering and knowledge management: Ontologies and the semantic Web. Springer, pp. 223-233.

Geonames (2014) http://geonames.org.

Goodchild, M. F. (2007) "Citizens as Sensors: The World of Volunteered Geography”. In GeoJournal, 69(4), p. 211-221.

Goodchild, M. F. and Glennon, J. A. (2010) "Crowdsourcing geographic information for disaster response: a research frontier". In Int. J. of Digital Earth, 3(3):231-241.

Google Maps API (2014) http://code.google.com/apis/maps/.

Haklay, M. and Weber, P. (2008) "OpenStreetMap: User-Generated Street Maps". In IEEE Pervasive Computing, 7(4): 12-18.

Haslhofer, B. et al. (2011) "The Open Annotation Collaboration (OAC) Model". In proc. of the IEEE Workshop on multimedia on the Web (MMWeb), pp. 5-9.

ITIL - Information Technology Infrastructure Library (2014) http://www.itilofficialsite.com/home/home.aspx.

Jena API (2013) http://jena.apache.org/documentation/ontology/index.html.

Kahan, J. and Koivunen, M. R. (2001) “Annotea: An open RDF infrastructure for shared Web annotations". In Proc. of the 10th Int. Conf. on World Wide Web, pp. 623-632.

Kim, H. L. et. al. (2008) "The State of the Art in Tag Ontologies: A Semantic Model for Tagging and Folksonomies". In Proc. of the Int. Conf. on Dublin Core and Metadata Applications, pp. 128-137.

Klien, E. and Lutz, M. (2005) "The role of spatial relations in automating the semantic annotation of geodata". In Spatial Information Theory, LNCS 3693, pp. 133-148.

Lamas, A. R. et al. (2008) "Sistemas de Informação Geográfica Móveis orientados ao contexto: uma abordagem baseada em ontologias de domínios". In Anais do IV Simpósio Brasileiro de Sistemas de informação (SBSI 2008), pp. 70-81.

Lorenz, B., Ohlbach, H. J. and Yang, L. (2005) "Ontology of transportation networks". In REWERSE Deliverable A1-D4, University of Munich, Institute for Informatics.

MapHub. (2013) "Maphub - Historic Map Annotation Portal". http://maphub.github.com.

Mayrhofer, A. and Spanring, C. (2010) "A Uniform Resource Identifier for Geographic Locations ('geo' URI)”. IETF RFC 5870. 
OpenLayers. (2014). http://docs.openlayers.org/.

OWL. (2004) "Web Ontology Language". http://www.w3.org/2004/OWL/.

Pedersen, J. et al. (2013). "Conceptual Foundations of Crowdsourcing: A Review of IS Research". In Proc. 46th Hawaii International Conference on System Sciences. 2013. pp.579-588.

PortoAlegre.cc. (2013) http://portoalegre.cc.

Prantner, K. (2004) "Ontour: The ontology. Technical report, DERI Innsbruck. http://etourism.deri.at/ont/docu2004/OnTour\%20-\%20The\%20Ontology.pdf.

Raimond, Y., Abdallah, S. (2007) "The Event Ontology". http://motools.sourceforge.net/event/event.html

Qureshi, H. et al. (2011) "Monitoring Disease Outbreak through Geographical Representation in Rural Areas". In Proc. of the Developments in E-systems Engineering (DeSE), pp. 30-35.

Sanderson, R. et al. (2013) "Open Annotation Core Data Model. W3C Community Draft". http://www.openannotation.org/spec/core.

Schulz, A. Ortmann, J. and Probst, F. (2012) "Getting User-Generated Content Structured: Overcoming Information Overload in Emergency Management”. In proc. of the Global Humanitarian Technology Conference (GHTC), pp. 143-148.

Sen, S. (2012) "Representing Geospatial Concepts: Activities or Entities?”. Universal Ontology of Geographic Space: Semantic Enrichment for Spatial Data. 2012.

Shah, et al. (2011) "Crowdsafe: crowd sourcing of crime incidents and safe routing on mobile devices". In: Proc. 19th ACM SIGSPATIAL Int. Conf. in Geographic Information Systems, p. 521-524.

Shaw, R., Troncy, R. and Hardman, L. (2009) "LODE: Linking Open Descriptions of Events". In Proc. of the 4th Asian Conf. on the Semantic Web, p. 153-167.

Ushahidi. (2013) http://www.ushahidi.com/.

Van Hage, W. R. et al. (2011) "Design and use of the Simple Event Model". In Web Semantics: Science, Services and Agents on the World Wide Web, 9(2):128-136.

W3C. (2003) "Basic Geo (WGS84 lat/long) Vocabulary". http://www.w3.org/2003/01/geo/wgs84 pos

W3Ca. (2013) "W3C Community and Business Groups". http://www.w3.org/community/openannotation/.

W3Cb. (2013) "Check and Visualize your RDF documents". http://www.w3.org/RDF/Validator/.

WGS84. (1984) "National Imagery and Mapping Agency: World geodetic system 1984”. Tech. Rep., National Imagery and Mapping Agency.

Zhao, P. et al. (2009) "Geospatial semantic web: critical issues". In Handbook of Research on Geoinformatics. 2009.

GONZALEZ, A. L.; IZIDORO, D.; WILLRICH, R.; SANTOS, C. A. S.

Representação Aberta e Semântica de Anotações Crowdsourcing em Mapas Web

iSys - Revista Brasileira de Sistemas de Informação, Rio de Janeiro, vol. 7, No. 2, p. 48-66, 2014. 